\title{
CFD Investigations of Effects of Cohesive Particles Proportion on Fluidization of Binary Particles
}

\author{
Liping Wei, Guodong Jiang, Haipeng Teng, Maosheng Zheng \\ School of Chemical Engineering, Northwest University \\ Taibai north road No.229, Xi'an, Shaanxi 710069, China \\ weiliping@nwu.edu.cn
}

\begin{abstract}
In this paper, numerical investigations of effects of cohesive particles proportion on the fluidization of binary particles was conducted by using Eulerain two flow model incorporating a new developed particle-particle drag model. Geldart-A and C particles consists the binary particles mixture. The bed expansion, mixing and segregation of the binary particles were simulated in different conditions of varying superficial velocity and Geldart-C particles proportion. It found that the particle-particle drag model can reasonably predicate bed expansion of the binary particles by introducing the parameter of solid surface energy to characterize the effect of cohesive collision energy loss. With an increase in superficial velocity, the bed voidage shows a nearly linear relationship vs. superficial velocity in logarithmic coordinates. Cohesive particles proportion greatly affects the mixing index of binary particles and optimal mixing is observed with increasing cohesive particles proportion at a certain superficial velocity. The mixing uniformity of binary particles is decreasing with an increase in superficial velocity.
\end{abstract}

Keywords: Fluidization, Cohesive particles, Particle-particle drag model, Mixing index, Binary particles

\section{Introduction}

The gasification of biomass in fluidized bed can produce clear energy for power plant and synthesis gas for further processing and obtaining high-value chemicals. The efficiency of gasification processes deeply depend on the effective regulations of fluidization of biomass particles. During the gasification, the biomass particles gradually convert to ash, which belongs to Geldart-C particles and easily adhere to other particles. Due to the high content of alkali metal, the ash has low-melting-point, which always induces lagging and leads to local defluidization. The complex distribution of multicomponent particles in the fluidized bed is an important influence factor for the gasification reactions. A reasonable evaluation of the effect of cohesive particles is necessary to predicate the distribution of the multi-competent particles.

The fluidization behaviour of binary particles deeply depends on the physical properties of cohesive particles (density and size etc.). It is not easy to accurately predicate the mixing or segregation characteristics of binary particles of cohesive powder under some operation conditions. Some CFD models have been established to describe the binary particles flow behaviours. The simulation of the fluidization processes of binary particle of Geldart-C and A or B can be conducted by Multiphase Emulation method by applying the particle-particle drag. There are two types of P-P drag in literature. One expression is developed by kinetic theory of granular flow, such as Chao et al.[1]. These methods connect the particleparticle drag coefficient to the average or individual granular temperature. The other way to derive the equation is based on an empirical voidage function, such as Bell[2]. Although the particle-particle drag models have been investigated widely, there are inconsistency of the impact of the particle-particle drag. However, the present particle-particle drag model can hardly be directly used to deal with cohesive particles flow because the effects of cohesive force during the collision process are not be considered when they are developed.

This work use a new derived particle-particle drag model for cohesive particles. The binary particle of cohesive particles and Geldart-A particles are simulated by applying the drag model to multiphase Eulerian model. 


\section{Simulation Model and Setup}

\subsection{Equations}

The model equations for the binary solid phases are investigated, thus the number of particle types is two. The index A and $\mathrm{B}$ represent the two types of particles. When the particles are fluidized, the slip velocity $\mathrm{u}_{-} \mathrm{sm}(\mathrm{s}=\mathrm{A}, \mathrm{B} ; \mathrm{m} \neq \mathrm{s})$ between the two particle phases induced the binary particle drag, which is considered as a dominant factor for the fluid dynamics. The control equations of Eulerian model are shown below, and index $\mathrm{g}$ and $\mathrm{s}$ refer to the gas and solid phase

The continuity equations of gas phase and solid phases are:

$$
\begin{gathered}
\frac{\partial}{\partial t}\left(\alpha_{g} \rho_{g}\right)+\nabla \cdot\left(\alpha_{g} \rho_{g} \vec{u}_{g}\right)=0 \\
\frac{\partial}{\partial t}\left(\alpha_{s} \rho_{s}\right)+\nabla \cdot\left(\alpha_{s} \rho_{s} \vec{u}_{s}\right)=0, s=A, B
\end{gathered}
$$

The momentum equations of gas phase and solid phases are

$$
\begin{gathered}
\frac{\partial}{\partial t}\left(\alpha_{g} \rho_{g} \vec{u}_{g}\right)+\nabla \cdot\left(\alpha_{g} \rho_{g} \vec{u}_{g} \vec{u}_{g}\right)=-\alpha_{g} \nabla p_{g}+\nabla \cdot \overline{\bar{\tau}}_{g}+\alpha_{g} \rho_{g} \vec{g}-\sum_{s=A, B} \beta_{g, s}\left(\vec{u}_{g}-\vec{u}_{s}\right) \\
\frac{\partial}{\partial t}\left(\alpha_{s} \rho_{s} \vec{u}_{s}\right)+\nabla \cdot\left(\alpha_{s} \rho_{s} \vec{u}_{s} \vec{u}_{s}\right)=-\alpha_{s} \nabla p_{g}-\nabla P_{s}+\nabla \cdot \overline{\bar{\tau}}_{s}+\alpha_{s} \rho_{s} \vec{g}+\beta_{g, s}\left(\vec{u}_{g}-\vec{u}_{s}\right)+\sum_{s=A, B ; m \neq s} \beta_{s, m}\left(\vec{u}_{m}-\vec{u}_{s}\right)
\end{gathered}
$$

Where $\overline{\bar{\tau}}_{g}$ is the viscous tress tensor of gas:

$$
\overline{\bar{\tau}}_{g}=\alpha_{g} \mu_{g}\left(\nabla \vec{u}_{g}+\vec{u}_{g}^{T}\right)-\frac{2}{3} \alpha_{g} \mu_{g} \nabla \cdot \vec{u}_{g} I
$$

The effective viscosity of gas phase $\mu_{g}$ includes two parts: dynamic viscosity $\mu_{g d}$ and turbulent viscosity $\mu_{g t}$, which can be determined by solving gas phase turbulence $k-\varepsilon$ models.

$$
\overline{\bar{\tau}}_{s}=\alpha_{s} \mu_{s}\left(\nabla \vec{u}_{s}+\vec{u}_{s}^{T}\right)+\alpha_{s}\left(\lambda_{s}-\frac{2}{3} \mu_{s}\right) \nabla \vec{u}_{s} I
$$

Where $\lambda_{s}$ is bulk viscosity

$$
\lambda_{s}=\frac{4}{3} \alpha_{s} \rho_{s} d_{s} g_{0, s s}\left(1+e_{s s}\right)\left(\frac{\Theta_{s}}{\pi}\right)^{1 / 2}, s=A, B
$$

The solid shear viscosity includes three parts: kinetic viscosity $\mu_{s, k i n}$ [3], collisional viscosity $\mu_{s, c o l}$, and frictional viscosity $\mu_{s, f}[4]$.

$$
\begin{gathered}
\mu_{s, \text { kin }}=\frac{10 \rho_{s} d_{s}}{96} \frac{\sqrt{\Theta_{s} \pi}}{\left(1+e_{i i}\right) g_{0, s s}}\left[1+\frac{4}{5} \alpha_{s} \rho_{s} d_{s} g_{0, s s}\left(1+e_{s S}\right)\right]^{2} \\
\mu_{s, \text { col }}=\frac{4}{5} \alpha_{s} \rho_{s} d_{s} g_{0, s s}\left(1+e_{s S}\right)\left(\frac{\Theta_{s}}{\pi}\right)^{1 / 2} \alpha_{s} \\
\mu_{s, f}=\frac{P_{s} \sin \Phi}{2 \sqrt{I_{2 D}}}
\end{gathered}
$$


$I_{2 D}$ is the second invariant of the deviatoric stress tensor. And $P_{S}$ is the solid pressure, $\Phi$ is the angle of internal friction. For binary particles, the solid pressure turns to [5]

$$
P_{s}=\alpha_{s} \rho_{s} \Theta_{s}+2\left(\frac{d_{s}+d_{m}}{2 d_{s}}\right)^{3} \rho_{s}\left(1+e_{s, m}\right) \alpha_{s} \alpha_{m} g_{0, s m} \Theta_{s}+2 \rho_{s}\left(1+e_{s m}\right) \alpha_{s}^{2} g_{0, s m} \Theta_{s} s=A, B ; m \neq s
$$

where, $g_{0, s m}$ is radial distribution function for binary mixture.

$$
\begin{gathered}
g_{0, s s}=\frac{1}{1-\frac{\alpha_{s}}{\alpha_{s, \max }}}+\frac{3}{2} d_{s}\left(\alpha_{m} / d_{m}+\alpha_{s} / d_{s}\right) \\
g_{0, s m}=\frac{d_{s} g_{0, s s}+d_{m} g_{0, m m}}{d_{s}+d_{m}}, \quad s=A, B ; m \neq s
\end{gathered}
$$

Where, $\alpha_{s, \max }$ is the max packed limit. The granular temperature equations are:

$$
\frac{3}{2}\left[\frac{\partial}{\partial t}\left(\alpha_{s} \rho_{s} \Theta_{s}\right)+\nabla \cdot\left(\alpha_{s} \rho_{s} \vec{u}_{s} \Theta_{s}\right)\right]=\left(-\nabla p_{s} I+\tau_{s}\right): \nabla \vec{u}_{s}+\nabla \cdot\left(k_{s} \nabla \Theta_{s}\right)-\gamma_{s}+\phi_{s m}, \quad s=A, B ; m \neq s
$$

The granular temperature $\Theta_{s}, k_{s}$ is the energy conductivity coefficient, and $\gamma_{s}$ is the collisional dissipation of energy fluctuation. The diffusion coefficient $k_{s}$ :

$$
k_{s}=\frac{150 \rho_{s} d_{s} \sqrt{\Theta_{s} \pi}}{384\left(1+e_{s s}\right) g_{o, s s}}\left[1+\frac{6}{5} \alpha_{s} g_{o, s s}\left(1+e_{s s}\right)\right]^{2}+2 \rho_{s} \alpha_{s}^{2} d_{s}\left(1+e_{s s}\right) g_{o, s s} \sqrt{\frac{\Theta_{s}}{\pi}}
$$

The rate of energy dissipation $\gamma_{s}$ :

$$
\gamma_{s}=\frac{12\left(1-e_{s S}^{2}\right) g_{o, s s}}{d_{s} \sqrt{\pi}} \rho_{s} \alpha_{s}^{2} \Theta_{s}^{3 / 2}
$$

The transfer of kinetic energy of random fluctuation between two types

$$
\phi_{s m}=-3 k_{s m} \Theta_{s}
$$

The gas-solid drag model is shown below [5]:

$$
\begin{gathered}
a_{g}<0.8 \beta_{g, s}=150 \frac{\alpha_{s}^{2} \mu_{g}}{\alpha_{g} d_{s}^{2}}+1.75 \frac{\rho_{s}\left|\vec{u}_{s}-\vec{u}_{g}\right|}{d_{s}} ; a_{g}>0.8 \beta_{g, s i}=150 \frac{\alpha_{s}^{2} \mu_{g}}{\alpha_{g} d_{s}^{2}}+\frac{3}{4} \frac{\alpha_{s} \alpha_{g} \rho_{s}\left|\vec{u}_{s}-\vec{u}_{g}\right|}{d_{s}} \alpha_{g}^{-2.65} \\
\text { where } C_{D}=\left\{\begin{array}{c}
\frac{24}{\alpha_{g} R e_{s}}\left[1+0.15\left(\alpha_{g} R e_{s}\right)^{0.687}\right], R e_{s} \leq 1000 \\
0.44, R e_{s} \geq 1000
\end{array}\right.
\end{gathered}
$$


The particle-particle drag coefficient for cohesive flow is [6]

$$
\begin{gathered}
\beta=\left(1+e^{\prime}\right) \frac{m_{i} m_{j}}{m_{0}} \frac{\alpha_{j}}{\frac{\pi}{6} d_{j}^{3} \frac{\alpha_{i}}{6} d_{i}^{3}}\left[\frac{\pi}{6}\left(d_{i}+d_{j}\right)^{3}+\frac{\pi}{4}\left(d_{i}+d_{j}\right)^{2} \lambda_{i j}\right] \frac{\left|\overrightarrow{\boldsymbol{c}}_{i j}\right|}{\lambda_{i j}} \\
e^{\prime}=\mathrm{e} \sqrt{1-\lambda_{c}} ; \lambda_{c}=\frac{2 m_{0} E_{c}}{m_{i} m_{j} e_{i j}{ }^{2} \vec{c}_{i j}^{2}} ; E_{c}=-\gamma \pi a^{2}
\end{gathered}
$$

Where, $\gamma$ is the solid surface energy per unit contact area, and $a$ is the contact area radius of the particles.

\subsection{Setup for Simulation}

The simulation parameters are shown in table 1 . The physical model of the fluidized bed is consistent with literature [7].

Table 1: Setup for simulation.

\begin{tabular}{|c|c|c|c|c|c|}
\hline Parameter & gas & FCC particles & $\mathrm{Al}(\mathrm{OH})_{3}$ & Parameter & Value \\
\hline $\begin{array}{c}\text { Density kg } \\
\mathrm{m}^{-3}\end{array}$ & 1.21 & 1659 & 2400 & Bed diameter $\mathrm{m}$ & 0.138 \\
\hline Viscosity $\mathbf{P a} \cdot \mathbf{s}$ & $1.78 \times 10^{-5}$ & & & Initial bed height $\mathrm{m}$ & 0.36 \\
\hline Diameter & & $60 \mu \mathrm{m}$ & $8 \mu \mathrm{m}$ & Coefficient of restitution & 0.9 \\
\hline Geldart group & & $\mathrm{A}$ & $\mathrm{C}$ & Grid $\mathrm{mm}$ & $0.1 \sim 0.5$ \\
\hline $\boldsymbol{u}_{\boldsymbol{m} \boldsymbol{f}} \mathbf{m} \cdot \mathbf{s}^{-\mathbf{1}}$ & & 0.0022 & & Time step & $10^{-5}-10^{-4}$ \\
\hline
\end{tabular}

\section{Results and Discussion}

Fig. 1shows the time-averaged voidage profile vs. superficial velocity. With an increase in superficial velocity, the voidage increases. The binary particles with $1 \mathrm{wt} \%$ cohesive particles shows a lowest bed expansion rate. The increasing cohesive particles proportion enhance the bed expansion rate. The cohesive particles may reduce the bed resistance by positing Geldart-A particle clearance. The cohesion effects between particles is not obvious when the bigger particles dominate the binary system. Fig. 2 shows the average fraction of cohesive particles vs. increasing superficial velocity and cohesive particles proportion. Both increasing superficial velocity and cohesive particles proportion reduce the solid fraction of cohesive particles. The slop of the profiles become large with an increase in cohesive particles proportion when the maximum fraction of Geldart-C below $15 \%$.

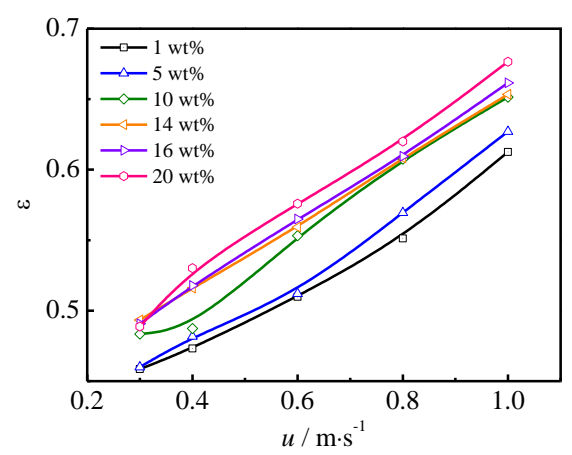

Fig. 1: Time-averaged voidage profile vs. superficial velocity. 


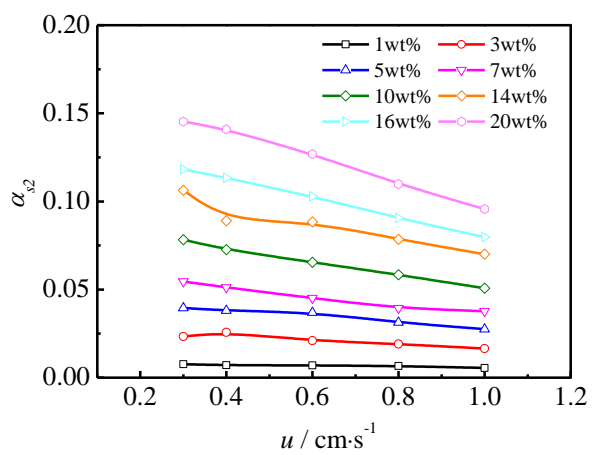

Fig. 2: Time-averaged Geldart-C solid fraction profile vs. $u$.

Fig.3 shows the effects superficial velocity on the mixability of binary particles. A mixing index $\mathrm{M}$ is defined as the ratio of the number of flotsam particles in mixing section to the total number of flotsam particles. With an increase in superficial velocity, the mixing index rapidly reduce. The increasing superficial velocity provides an enhanced gas-solid drag force, which increase the segregation of Geldart-A and C particles within the fluidized bed. Fig. 4 shows the effects of weight proportion of cohesive particles on the mixing index. It can be observed that an optical mixing can be obtained at a certain value of wt, the mixing index reduce above or below the value, which may attributed an competition between gassolid interaction and particle-particle interaction.

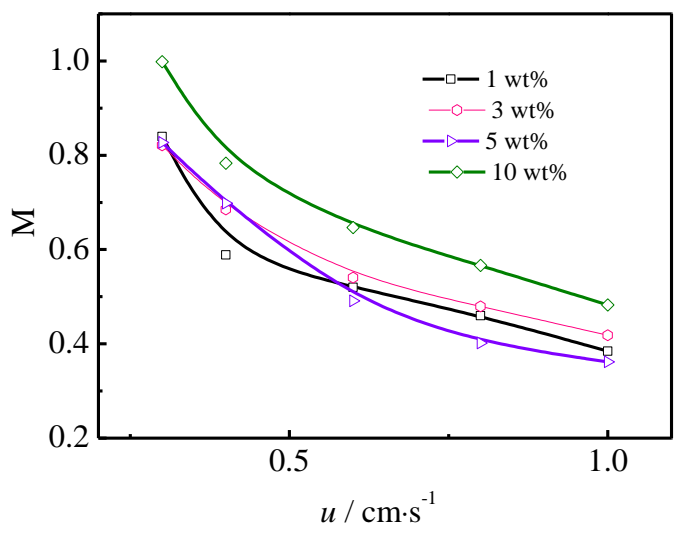

Fig. 3: Variation of mixing index vs. superficial velocity.

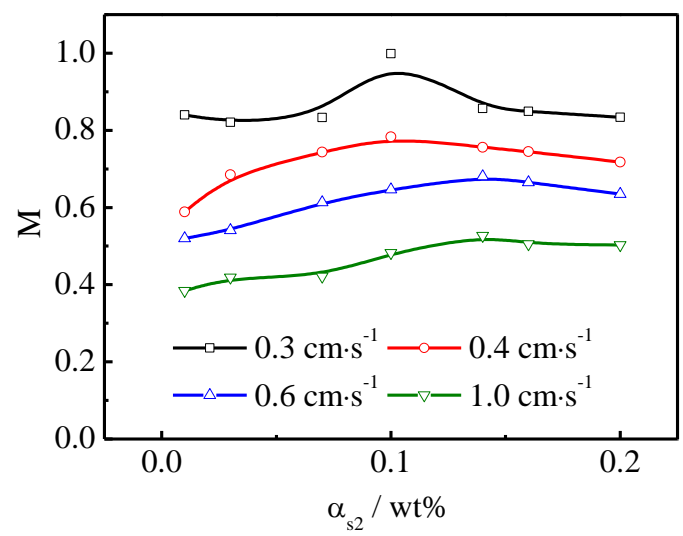

Fig. 4: Effects of cohesive particles proportion on the mixing index. 


\section{Conclusion}

The conclusions of the CFD investigations include: (1) the particle-particle drag model can reasonably predicate bed expansion of the binary particles; (2) with an increase in superficial velocity, the cohesive particle fraction and mixing index reduce; (3) cohesive particles proportion greatly affects the mixing index of binary particles and optimal mixing is observed at a certain cohesive particles proportion.

\section{Acknowledgements}

This work is currently supported by the National Natural Science Foundation of China through contract No.51606153, Youth Science Foundation of NWU (15NW15) and Natural Science Basic Research Plan in Shaanxi Province of China (No. 2016JQ5101).

\section{References}

[1] Z. Chao, Y. Wang, J. P. Jakobsen, M. Fernandino, H. A. Jakobsen, "Investigation of the particle-particle drag in a dense binary fluidized bed," Powder Technology, vol. 224, no. 7, pp. 311-322, 2012.

[2] R. A. Bell, "Numerical modelling of multi-particle flows in bubbling gas-solid fluidised beds," Licentiate thesis, Melbourne: Swinburne University of Technology, 2000.

[3] J. Ding, D. Gidaspow, "A bubbling fluidization model using kinetic theory of granular flow," AIChE Journal, vol. 36, no. 4, pp. 523-538, 1990.

[4] D. G. Schaeffer, "Instability in the Evolution Equations Describing Incompressible Granular Flow," J. Diff. Eq., vol. 66, pp. 19-50, 1987.

[5] D. Gidaspow. Multiphase Flow and Fluidization. Boston: Academic Press, 1994.

[6] L. Wei, "Particle-particle drag model for cohesive particles flow-model derivation and validation," Annual meeting of the multiphase flow, Guangzhou, China, 2016.

[7] T. Nakazato, Y. Suzuki, E. A. Mahmoud, et al., "Effect of Size and Hold-Up of Cohesive Fine Powders on Particulate Fluidization of Binary Powder-Particle Mixtures," Asian Pacific Confederation of Chemical Engineering Congress Program, 2004. 